$\xi=-1$

\title{
Implementation of Internet of things in a mobile Humanoid robot: A base for future of IOT enabled robotics applications
}

\author{
D Jalamkar ${ }^{1}$, Krishnakumar $^{2}$, Arockia Selvakumar A*3 \\ ${ }^{1,2,3}$ Vellore Institute of Technology, Chennai, Tamil Nadu, India \\ *Corresponding author E-mail: arockia.selvakumar@vit.ac.in
}

\begin{abstract}
Internet of Things (IoT) is the most growing and trending technology recently in the field of mechatronics engineering. Main reason behind the growth of this technology is that it can be implemented to almost everything and everywhere. Internet of things has pretty much evolved from the "Next big thing" to the "biggest thing" happening around us right now. In this paper, a complete analysis is provided to understand the amalgamation of robotics and internet of things. Robotics and Internet of things are two big fields where successive research is happening. The integration of these two would make the monitoring, implementation way better. Hence keeping this in mind, a complete analysis is made, with the hardware, software, platforms available also taken into consideration. Also other factors such as simplicity, cost, and ease of development are taken into consideration.
\end{abstract}

\section{Keywords: Internet of Things; Humanoid robot; control; automation}

\section{Introduction}

In general, in the present day, humanoid robots are being used as domestic robots in houses as well as in industries. But the domestic ones are quite common in the recent trend. And when we look at the Internet of things, it has been spreading across all fields, and making systems easier. If we take the case of domestic robots, home security is one key role given to them. Intruder detection, taking care of visitors, taking safety measures in case of disastrous accidents and so on. If the robot acts as an independent system, then the user won't be able to check or monitor the various activities carried out. He'll be able to see the physical output and that will be it. But in the case of Internet of things linked up with such a robot, the whole process becomes hassle free. The user will be able to check the status through his mobile application from elsewhere, he can get an alert in case of an intruder, an alert in case there is a chance of accident inside the house and using these data, he will easily be able to make the robot act accordingly by controlling it through the app itself. As we can see, the combination of these two revolutionary technologies has the potential to make every job easy and almost everything accessible for human beings.

When we start looking into the Internet of things, a lot of data transfer takes place between various things and the server because basically Internet of things is primarily about connecting multiple devices by using internet and let them interact with each other. So basically the speed of internet connectivity does matter a lot. With the $5 \mathrm{G}$ technology rising into the picture, the whole idea of Internet of things becomes even more suitable. It is predicted that around 50 billion things will be connected and thus making the Internet of things more efficient. It would also make it faster and effective. IoT is being used in so many industries such as the healthcare industry and industrial plants. And now it comes into the picture of robots. Connecting many robots across the world wherever there is internet connectivity and hence making a very useful network. This comes up to a new term - 'The internet of robotics things' shortly known as the IoRT. And this branch of
IoRT can be implemented into various places and we are going to be looking at the domestic humanoid application of IoRT.

\section{Recent IoT Hardwares available}

\subsection{Choice of Development board:}

Right now Arduino is one of the most used Microcontroller development boards which can pretty much cover up controlling all the aspects of a domestic humanoid robot. Most of the hardware is compatible with Arduino and a lot of Arduino compatible devices are available in the market. It also has a lot of libraries which are available online which makes coding easier in the IDE. And when it comes to Internet of things, Arduino is kind of very much suited as there are numerous options available to be chosen based on the application and requirement. Other advantages include the small size, cost effectiveness and low power consumption.

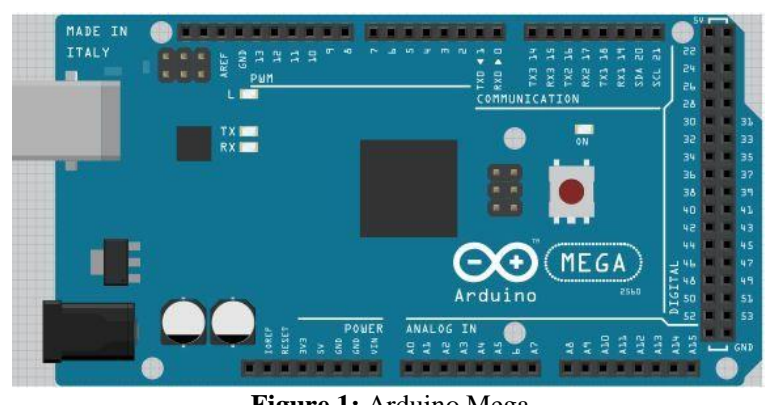

Figure 1: Arduino Mega

\subsection{WiFi Module}

This major disadvantage of the other possible modules such as the GSM module, Ethernet shield, Zig Bee could be avoided by making used of an Internet connection to a WiFi router and

Copyright $\odot 2018$ Authors. This is an open access article distributed under the Creative Commons Attribution License, which permits unrestricted use, distribution, and reproduction in any medium, provided the original work is properly cited. 
creating a hotspot for the ESP8266/ WiFi module. Various versions of ESP 8266 come in different configurations for different applications. They vary in terms of number of GPIOs, cost, size, processing speed, memory, etc [12]. WiFi module like ESP 8266 is recently developed hardware which is updated from version 1 to the latest version 14 . This is very cheap module with system on chip. ESP 8266 can work independently without connecting to any microcontroller or microprocessor. ESP 8266 version 01(as shown in fig.2.) is the most widely used module for small applications where less GPIOs are needed. This is the low cost version available today. This hardware is the most promising in terms of connecting anything to the internet.

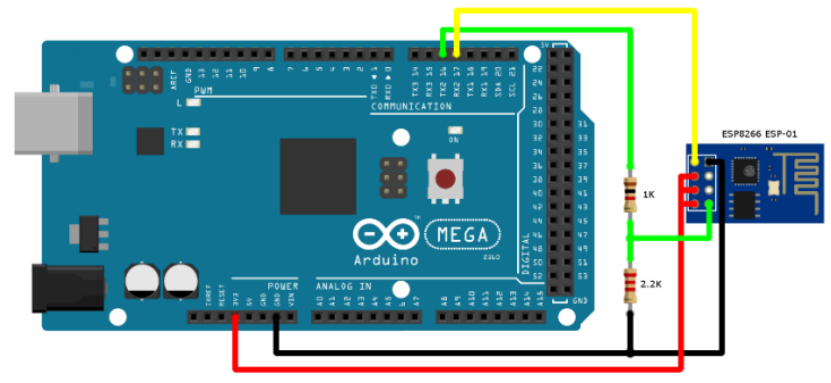

Figure 2: ESP8266 with Arduino Mega

\section{Protocols for IoT}

MQTT is very suitable for remote location messaging and it has a publish-subscribe architecture. In this architecture, the sensors and the actuators are connected to a server or broker[13]. The message to the server is done in the form of chunk. This data is published to the connected clients by the server. Mostly used for wireless sensor networks, more particularly for embedded systems. The general concern of security is also taken care of. It asks for a username and a password to connect to the broker. The 5 ways in which the MQTT protocol works will be connecting to the server, disconnecting once the task is done, waiting for subscribing the client to the broker, unsubscribe from connected things and publish - display the values to the client.

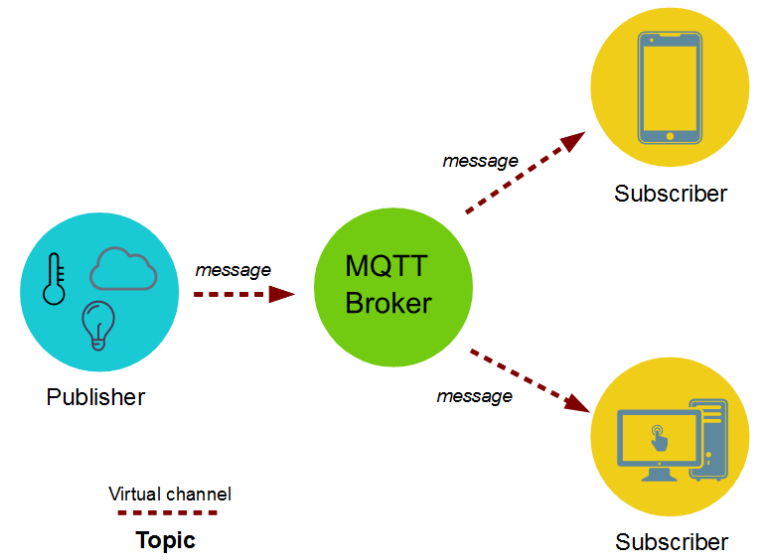

Figure 3: MQTT Flow

CoEP is particularly made for machine to machine interaction which is a key aspect of IoT. It is basically based on UDP and UDP makes sure that the hardware quickly wakes up. It transmits small packets of data with low overhead and this makes the connected things to hold in sleep mode for a longer time and hence saving the battery. CoAP is kind of similar to HTTP but MQTT is more stable and reliable as it is easy to get up in working network. Taking these factors into consideration, MQTT is the best option.

\section{Hardware Selection and Implementation}

From the various hardware compared above, considering various advantages and other factors, ESP8266 version $12 \mathrm{WiFi}$ modules was chosen for the project. Along with the WiFi module, Arduino Mega microcontroller was chosen because of large number of GPIOs. A humanoid robot with 14 degrees of freedom was fabricated for the project. The humanoid robot consisted of $12 \mathrm{RC}$ servo motors at various joints on arms, grippers and head. Forward and backward movement to the robot was given by using two high torque DC motors. The various hardware components used for the humanoid robot is shown in Table 1.

Table 1: Hardware components

\begin{tabular}{|l|l|}
\hline Component & Quantity \\
\hline Arduino mega board & 1 \\
\hline ESP8266-12 WiFi modules & 2 \\
\hline DC Motor driver board & 1 \\
\hline 30kgcm servo motors for arms & 6 \\
\hline $\begin{array}{l}1 \mathrm{~kg} \mathrm{~cm} \text { servo motors for gripper } \\
\text { and head }\end{array}$ & 4 \\
\hline $\begin{array}{l}110 \mathrm{~kg} \mathrm{~cm}, 10 \mathrm{rpm} \text { DC motors for } \\
\text { wheels }\end{array}$ & 2 \\
\hline $12 \mathrm{~V} 5 \mathrm{amp}$ DC Adapter & 1 \\
\hline LM35 Temperature sensor & 1 \\
\hline
\end{tabular}

Figure 4 shows the system layout of the project. A smart phone connected to the internet is used to control the robot. An android application was made with a user interface that is required to control the robot. Different widgets are added to the user interface for different tasks [18]. Smartphone is connected to the MQTT Broker by using the key provided by the broker. Here, Cloudmqtt.com is used as a broker for IoT.

On the other side, Two ESP8266 version $12 \mathrm{WiFi}$ modules are paired to the same server by using the same key provided by the broker. Two modules are used in order to increase the number of GPIOs. WiFi modules are connected to the internet by connecting them to any WiFi hotspot having some SSID and password.

For the motion control of the arms, head and gripper, one slider for each motor is added to the android application user interface to send PWM signals to the WiFi module via MQTT broker. Sliders are used to produce the PWM signals. WiFi modules receive the control signals from the WiFi router with internet connectivity. As shown in fig.9. Two WiFi modules are further connected to the Arduino mega microcontroller. Arduino microcontroller is used to scale the PWM signals coming from the WiFi module to the desired range of angle in degrees. For the $0 \%$ to $100 \%$ motion of a slider, servo motor rotates from $0^{\circ}$ to $180^{\circ}$ angle. A default initial value for each motor according to the need of joint is set in the Arduino program, so that on start up all the motors will be positioned to the desired default value. 


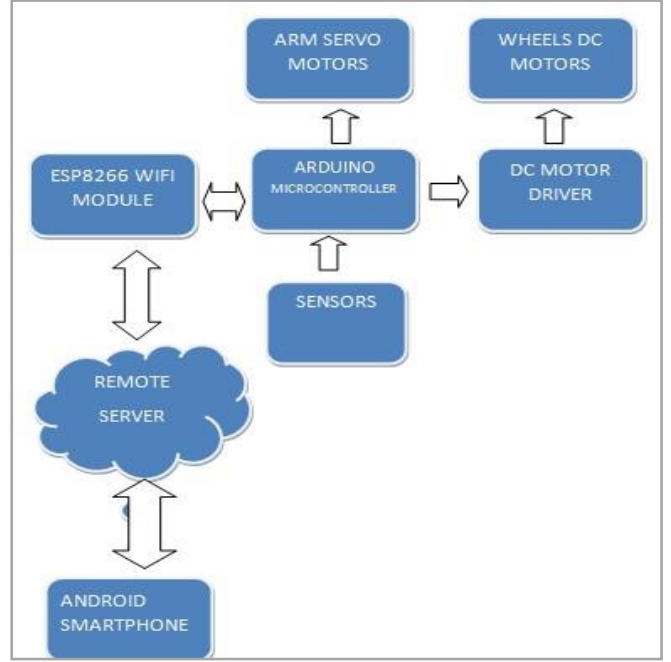

Figure 4. Block Diagram of Proposed Control System

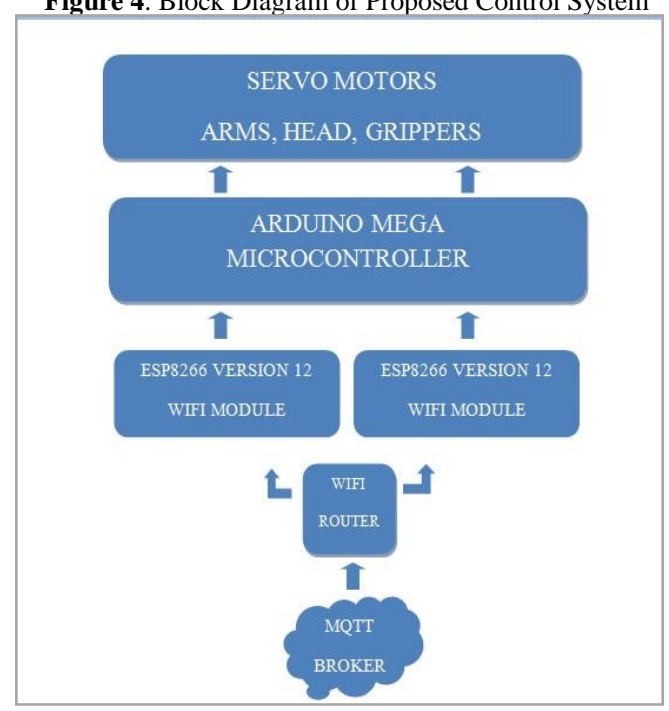

Figure 5. Servo Motor control

For the motion control of the arms, head and gripper, one slider for each motor is added to the android application user interface to send PWM signals to the WiFi module via MQTT broker. Sliders are used to produce the PWM signals. WiFi modules receive the control signals from the WiFi router with internet connectivity. As shown in fig.9. Two WiFi modules are further connected to the Arduino mega microcontroller. Arduino microcontroller is used to scale the PWM signals coming from the WiFi module to the desired range of angle in degrees. For the $0 \%$ to $100 \%$ motion of a slider, servo motor rotates from $0^{\circ}$ to $180^{\circ}$ angle. A default initial value for each motor according to the need of joint is set in the Arduino program, so that on start up all the motors will be positioned to the desired default value.

Four toggle switches are added to the android application user interface to send $\mathrm{HI} / \mathrm{LOW}$ signals to the WiFi module via MQTT broker. Also, programming for forward, backward, left, right movements of robot is done in Arduino. For these movements, Arduino gets only high/low signals from the WiFi modules which is then converted to the specific commands by Arduino and are given to the dc motor driver and them ultimately to the dc motors. These two dc motors drives the wheels in order to achieve the motion. Along with these actuators, a temperature sensor LM35 was directly connected to one of the WiFi module on its ADC pin with external power supply. Modelling of the frame was done in the CATIA V5, according to the model; body was fabricated in the workshop with slight necessary modifications.
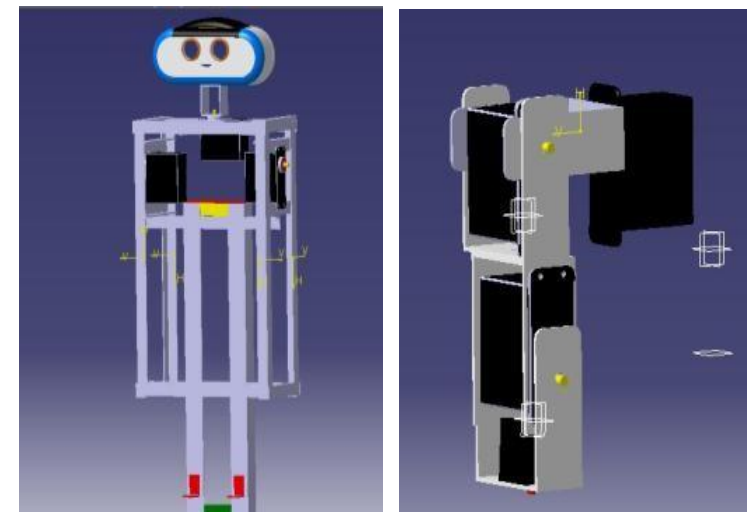

Figure 6. CATIA V5 model of the frame and arm [19]

For the body of a humanoid robot, Aluminium material was selected because of its high strength and low weight. Density of aluminium is one third of that of steel. Aluminium is easy to fabricate as compared to other materials. L sections of aluminium are used to ensure the strength and stability.

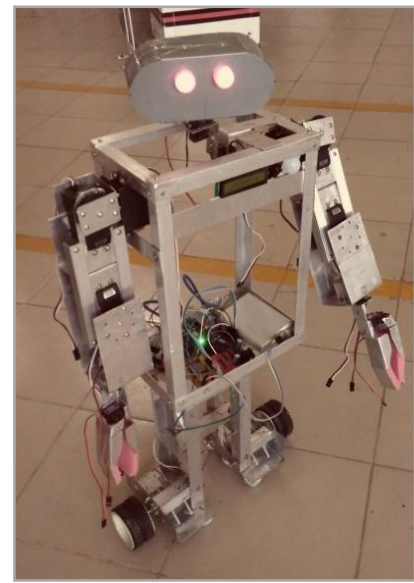

Figure 7. Hardware setup

According to the model, body was fabricated in the workshop with slight necessary modifications. Frame was fabricated with L sections with dimensions 2 inch and 2.5 inch and $1 \mathrm{~mm}$ thickness. For arms, $0.8 \mathrm{~mm}$ thick aluminium sheet metal was used.

\section{Results and Discussion}

Three prototypes of the gripper were fabricated for the robot. Each was tested and examined whether they are suitable for the robot or not. Accordingly following results were obtained. Since solenoid pulls the iron core completely inside the tube, it is difficult to hold the iron code in intermediate position in order to half open or half close the gripper fingers to grasp various objects with different sizes. Solenoid has only ON and OFF positions and it gets heated very fast if the current drawn is more. In order to increase the gripping force, the size of the coil increases because of that the weight, which is not desirable. After testing the screw gripper there were some problems encountered with it. Difficult to fabricate this type of gripper in smaller size because of more number of links and complex structure. After grasping the object, motor keeps rotating so it needs a limit switch to stop at certain position which is difficult to execute and occupied extra GPIOs on the controller. So it is undesirable to use this gripper after the above difficulties. Also actuation of this gripper is very slow. In order to get more torque, speed of the motor should be low which ultimately results in slower actuation. Each gripper can be opened wide up to 180 degree angle. This angle can be controlled by setting the opening limit in the Arduino program. Default position 
of the motor is kept at 0 degree angle so the gripper remains close normally. Gripper can be opened by moving the gripper slider in the android application. $0 \%$ on the slider corresponds to the fully closed gripper at 0 degree motor rotation, while $100 \%$ on the slider 37 corresponds to the fully open gripper at 180 degree motor rotation. Also from $0 \%$ to $100 \%$ any middle position is possible.

Using the android application, multiple actuators and sensors were connected to the controller - Arduino Mega which in turn was connected to the WiFi by using ESP8266-12. For this, PWM signal was generated by using a slider widget in the android application. Further this signal was transmitted to the WiFi module via MQTT broker. From WiFi module the signal is given to the Arduino microcontroller for scaling into required degrees of angle of rotation. By using CloudMQTT server for MQTT protocol connection, the connection time required to send each command was observed to be less than a second which still varies with some unavoidable factors such as server traffic and internet connection speed. While subscribing to the MQTT broker service, a paid plan can be selected to get more bandwidth and more number of connections to connect more actuators at a time. These services can be subscribed on monthly payments.

The bot was actuated based on the signals sent from the android application. It was observed while using the sliders on android application, only one motor at a time was able to actuate. If multiple sliders are moved at a time, the commands are queued and are sent to the server one by one. User interface in the android app is made by using ionic framework and can be edited by editing the HTML code in the WiFi module. Various widgets can be added to the user interface to perform various tasks.

After the testing, following difficulties were faced:

Only ON/OFF control was possible.

Very slow server speed. (10 to 30 seconds per action)

Connectivity issues were observed.

\section{Conclusions}

In the picture of IoRT (Internet of Robotic Things), it is very important to identify the tasks that are to be done by the robot. Only this will be able to give a proper clarity as to which module or protocol or hardware to use where. For instance, it requires many actuators, mostly DC motors for movement and Servos for arm actions and these require quite a lot of GPIO pins and thus highlighting the need to select Arduino Mega in our case. Also we discussed about the Wemos D1 board which could have been used as an alternative to the ESP 8266 as the module was giving initialization problems in the beginning. Also we found that there were a few connectivity issues and that only one command that could be sent at a time. This method was used along with the MQTT protocol for communicating which is one of the most stable protocols for the Internet of things. Pros and cons of various methods, hardware, architectures, protocols for IoT should be considered in order to make the proper selection depending upon the need of application. Implementation of IoT to the humanoid robot can be taken to very sophisticated levels in the future. IoT enabled humanoid robots can be used in many different sectors where there are not used till the date. Man to robot interaction can be taken to the next advanced level with further developments in the area of Internet of Things. This technology is going to push the limits of modern Engineering and create a revolution. Looking towards the processing and controlling needs, Arduino microcontroller is used in the project. In order to increase the processing and 41 controlling capabilities of the robot, an advanced microcontroller or even microprocessors like raspberry pi, etc. can be used further. In order to make hardware modifications, more sophisticated servo motors can be used which will restrict back emf so as to save the microcontroller circuits. Currently high torque servo motors are struggling to restrict the back currents are damaging the microcontroller. Low torque servo motors are working fine without any difficulty. Use of gyroscope can be made in order to improve the balancing. Also the 3D printed parts can be attached in order to improve the external looks and appearance. Use of Wemos D1 board could be done to reduce the complexity.

\section{References}

[1] Leslie D'Monte "IoT is not hype, but it's also not some magic technology: Sanjay Sarma" http:/ www.livemint.com http://www.livemint.com/ Companies BJyX5OJCizP79P5uCX6tRO / IoT-isnot-hype-but- its-also-notsome-magic-technologySa.html

[2] Deb Miller Landau "How 5G will Power the Future Internet of Things" http://iq.intel.in http://iq.intel.in/how-5g-will-power-thefutureinternet-of-things.

[3] Udit Roy "Internet of Things : Let devices do the talking" http://indianexpress.com http://indianexpress.com/article/technology/technewstechnology/internet-of-things-let-devices-dothe-talking

[4] Grieco, L. A., A. Rizzo, S. Colucci, S. Sicari, G. Piro, D.DiPaola, and G. Boggia (2014). "IoT-aided robotics applications: Technological implications, target domains and open issues." Computer Communications 54 (2014): 32-47.

[5] Buschmann, Thomas, Sebastian Lohmeier, and Heinz Ulbrich(2009). "Humanoid robot lola: Design and walking control." Journal of physiology-Paris 103, no. 3 (2009):141-148

[6] Dmitri shiryaev "RFID technology and internet of things "http://www.slideshare.net http://www.slideshare.net/rushtek/rfidand-internet-ofthings

[7] Ray Floyd "RFID and the Internet of Things" http://www.engineering.com

http://www.engineering.com/ElectronicsDesign/Electr onicsDesignArticles/ArticleID/8145/RFID-and-theInternet-ofThings.aspx

[8] Kadir, Wan Muhamad Hanif Wan, Reza Ezuan Samin, and Babul Salam Kader Ibrahim(2012). "Internet Controlled Robotic Arm." Procedia Engineering 41 (2012): 1065- 1071

[9] Charalampos Doukas, "Building Internet of Things with the Arduino" www.BuildingInternetofThings.com

[10] Qu, Liguo, Yourui Huang, Chaoli Tang, and Tao Han (2012)."Node design of internet of things based on Page 7 of 7 ZigBee multichannel." Procedia Engineering 29 (2012): 1516-1520

[11] "Getting Started with the Arduino GSM Shield" www.Arduino.cc https://www.Arduino.cc/en/Guide/ArduinoGSMShield

[12] "ESP8266-module-family" http://www.ESP8266.com http:// www.ESP8266.com/wiki/ doku.php?id = ESP8266-module-family

[13] Toby Jaffey "MQTT and CoAP, IoT Protocols" eclipse.org https://eclipse.org/community/eclipse_newsletter/2014 /february/article2.php

[14] "Protocol Specifications" mqtt.org http://mqtt.org/documentation

[15] "MQTT" Wikipedia page https://en.wikipedia.org/wiki/MQTT

[16] Carsten Bormann "RFC 7252 Constrained Application Protocol" Homepage, http://coap.technology/

[17] James stansberry "MQTT and CoAP: Underlying Protocols for the IoT" electronicdesign.com http://electronicdesign.com/iot/mqttand-coapunderlying- protocols-iot

[18] "Ionic Documentation Overview" http://ionicframework.com http://ionicframework.com/docs/ overview/

[19] D Jalamkar and Arockia Selvakumar A (2017), Use of internet of Things in a Humanoid Robot - A Review, Advances in Robotics and Automation Journal, doi.10.4172/2168-9695.1000149. 